\title{
Transatlantica
}

Revue d'études américaines. American Studies Journal

\section{Colloque international « Infinite Wallace/ Wallace Infini »}

Universités Paris 3-Sorbonne Nouvelle et Paris 7-Paris Diderot, 11-13

septembre 2014

\section{Ariane Mak}

\section{(2) OpenEdition}

\section{Journals}

Electronic version

URL: https://journals.openedition.org/transatlantica/7086

DOI: $10.4000 /$ transatlantica.7086

ISSN: $1765-2766$

Publisher

Association française d'Etudes Américaines (AFEA)

Electronic reference

Ariane Mak, "Colloque international «Infinite Wallace/ Wallace Infini »", Transatlantica [Online], 2 | 2014, Online since 05 March 2015, connection on 31 January 2023. URL: http://journals.openedition.org/ transatlantica/7086 ; DOI: https://doi.org/10.4000/transatlantica.7086

This text was automatically generated on 31 January 2023.

Creative Commons - Attribution-NonCommercial-NoDerivatives 4.0 International - CC BY-NC-ND 4.0 https://creativecommons.org/licenses/by-nc-nd/4.0/ 


\section{Colloque international « Infinite Wallace/ Wallace Infini »}

Universités Paris 3-Sorbonne Nouvelle et Paris 7-Paris Diderot, 11-13 septembre 2014

\section{Ariane Mak}

1 C'est dans le contexte d'un développement important des études wallaciennes en Europe et en Amérique du Nord que les Universités Paris 3-Sorbonne Nouvelle et Paris 7-Paris Diderot ont organisé une conférence internationale autour de l'écrivain américain David Foster Wallace (1962-2008) les 11, 12 et 13 septembre 2014 à Paris.

2 À travers ses romans - Infinite Jest (1996) en tête - ses nouvelles, écrits philosophiques et journalistiques, David Foster Wallace s'est imposé comme l'un des plus talentueux auteurs de sa génération. Pourtant, en France, le grand public comme le milieu de la recherche n'ont pas suivi cette œuvre d'une manière significative. Béatrice Pire (Paris 3), Pierre-Louis Patoine (Paris 3) et Mathieu Duplay (Paris 7) sont à l'origine de ce colloque international qui entendait éveiller un nouvel intérêt pour l'œuvre wallacienne et en présenter les approches critiques les plus récentes.

3 Douze panels rassemblant trente-six intervenants ${ }^{1}$ ont exploré des problématiques variées: "Performance, entertainment, media ", "Wallace the auteur/Questions of reading and writing ", "Influences and transmissions ", "Post-secular Wallace?", « David Foster Wallace and Philosophy », « Humor, Sentiment, Communion ».

\section{De nouvelles directions pour les études wallaciennes}

Les trois jours de ce colloque international ont été l'occasion de découvrir les approches inédites empruntées par des études wallaciennes en profond renouvellement. Pour commencer, le colloque a mis en évidence l'élargissement des thématiques à l'œuvre dans les Wallace Studies. De fait, l'analyse critique des essais et écrits journalistiques de Wallace, jusque-là négligés au profit de son œuvre de fiction, a mis au jour des problématiques nouvelles relevant de la musique, de la religion ou du situationnisme. D’autre part, le dépôt des archives de David Foster Wallace au Harry 
Ransom Center d'Austin a changé la donne pour l'étude des écrits de Wallace. Lee Konstantinou a montré que ces archives permettaient des analyses du processus d'écriture de Wallace, et ouvrait la voie à une critique génétique de son œuvre. Un mouvement qui s'accompagne d'ailleurs d'un déplacement de la focale vers le paratexte de l'œuvre wallacienne : analyse des marginalia pour Mike Miley, étude des logiques de présentation des éditeurs et quatrièmes de couverture pour Tore Andersen.

5 Les chercheurs rassemblés ont en outre pointé les angles morts de la discipline, et appelé de leurs vœux l'émergence d'études consacrées à ces problématiques négligées. Ainsi, pour Mary Holland, les Wallace Studies sont entrées dans une nouvelle période qui autorise une étude plus critique de l'œuvre de Wallace. Elle a en outre déploré l'absence quasi totale d'analyses prenant en charge les notions de race, de classe et de genre dans ses écrits. Adam Kelly a quant à lui proposé d'opérer un renversement important : ne plus nous demander ce que des disciplines diverses (musique, mathématiques, queer theories,...) ont à nous dire sur l'œuvre de Wallace, mais ce que celles-ci peuvent leur apporter.

6 Suivent des plans rapprochés sur cinq communications - un échantillon qui, en dépit de sa taille réduite, entend donner une idée de la richesse des interventions entendues lors du colloque.

\section{Session plénière. Marshall Boswell (Rhodes College, Memphis, USA), " The Wallace Effect: the Anxiety of Wallace's Influence in Contemporary Fiction »}

7 S'emparant de The Marriage Plot (2011) de Jeffrey Eugenides, Marshall Boswell a analysé la présence spectrale de Wallace dans la fiction américaine contemporaine. L'intervention a démontré que Leonard Bankhead, personnage clé de The Marriage Plot, était fortement inspiré de David Foster Wallace. On lui retrouve le bandana, attribut wallacien par excellence, une forte consommation de tabac à chiquer et une apparence de "gentil géant " alliée à une délicatesse de l'expression. Mais la ressemblance ne s'arrête pas là. Certaines phrases prononcées par Wallace sont reprises verbatim et attribuées à Leonard Bankhead, et les nombreuses références à Werther constituent autant de clins d'œil au surnom de «Young Werther » que se donnait Wallace dans sa correspondance avec Mary Karr.

8 En invoquant ainsi la présence de Wallace, Eugenides fait de son livre une allégorie ambiguë de la relation entre le post-postmodernisme et la fiction post-moderne qui l'a précédé. La contribution a souligné qu'il ne s'agissait pas d'un simple hommage à Wallace, dans le sens où The Marriage Plot esquisse une critique de la réception des œuvres de Wallace d'une part, et ouvre un espace métafictionnel dialogique entre les deux auteurs d'autre part. En définitive, Marshall Boswell a proposé de considérer The Marriage Plot comme un triangle amoureux dans lequel Wallace et Eugenides se disputent l'attention du lecteur. 


\section{Laura Kreyder (Université de Milan, Italie), « Le bon usage : le français chez David Foster Wallace»}

Nombreux sont les lecteurs francophones à s'être interrogés devant l'utilisation fautive de la langue française dans Infinite Jest (1996). Celle-ci paraît d'autant plus étonnante de la part de Wallace, fervent défenseur de la linguistique prescriptive. L'intervention de Laura Kreyder a démontré qu'il fallait y voir une stratégie littéraire privilégiant la charge comique de la traduction. Ainsi les terroristes québécois d'Infinite Jest, reconnaissables à leurs chaises roulantes, ont-ils été affublés de noms divers au fil du processus d'écriture. Les « Assassins des Voitures de Malade » pour commencer ; «Les Assassins des Fauteuils Roulents " ensuite, dans un français quasi correct; "Les Assassins des Fauteuils Rollents " pour finir. C'est donc l'allitération reproduisant le roulement, et une parodie de la langue française pour les non-francophones, qui ont été privilégiées plutôt que la justesse de la langue. Plusieurs exemples de jeux de mots bilingues ont en outre été cités, parmi lesquels «toot sweet " pointant vers le français «tout de suite» ou le «Minister of Environment and Resources Development Enterprise » à l'acronyme potache.

S'appuyant sur l'examen de la bibliothèque de Wallace conservée à Austin, l'intervention a également retracé l'influence de la culture française sur l'œuvre de Wallace. Ainsi a-t-on découvert que Wallace avait lu Rabelais, Stendhal, Chrétien de Troyes et Gide alors qu'il était étudiant à Amherst. Il possédait également un exemplaire de L'Amant de Duras (1984), de l'ouvrage de Nathalie Charraud sur Cantor (1994) ainsi que L'Histoire politique du barbelé d'Olivier Razac (2000), dont on retrouve l'influence dans The Pale King (2011). Camus, Rousseau, Artaud et Pérec font en outre partie des auteurs mentionnés dans Infinite Jest. Laura Kreyder a aussi nuancé l'idée très répandue dans les Wallace studies, selon laquelle Wallace avait été un avide consommateur de la culture cinématographique française. Wallace semble en réalité avoir moins vu que lu sur le cinéma français, a affirmé Laura Kreyder, avançant que $L a$ Sentinelle (1992) de Desplechin était le seul film français dont on puisse être certain qu'il avait été vu par Wallace.

\section{Christopher Kocela (University of Georgia State, Atlanta, USA), « Engaging Buddhism in the Work of David Foster Wallace: Continuous Practice, Infinite Jest»}

11 L'une des plus surprenantes interventions du colloque a été celle de Christopher Kocela qui explorait un thème a priori éloigné de l'univers wallacien : celui du bouddhisme zen. Christopher Kocela a souligné l'étonnante récurrence des comparaisons au bouddhisme dans les écrits de Wallace concernant le sport. Dans « Derivative Sport in Tornado Alley » (1997), Wallace mentionne ainsi «my Zen-like acceptance of things as they were on court ». Dans « A Supposedly Fun Thing I'll Never Do Again », il affirme: «3P Winston and I have both reached that level of almost Zen-like Ping-Pong mastery where the game kind of plays us ». Quant au Coach Schtitt d'Infinite Jest, ne conseille-t-il pas aux jeunes joueurs de tennis de voir le filet et leur adversaire comme des alliés dans une quête de transcendance personnelle? 
12 La contribution a proposé une réinterprétation du rapport de Wallace à la méditation et démontré l'importance des motifs bouddhistes dès ses premiers essais journalistiques comme dans Infinite Jest. En cela, Christopher Kocela a remis en cause le paradigme selon lequel la fiction wallacienne s'était déplacée d'une critique de l'ironie (avec «E Unibus Pluram », (1997)) vers des questionnements liés à la spiritualité et à la contemplation («This is Water » (2009)).

\section{Tore Andersen (Arhus University, Danemark), "Covered in "P" - David Foster Wallace's Paratextual Curse »}

13 «I bristle sometimes at getting compared to [...] these classic postmodern guys. The the - the "P" guy comes into mind. I won't even say his name »: voilà ce que répondait Wallace à une interview en 1997. Comment, en effet, Thomas Pynchon est-il devenu la référence systématique à laquelle Wallace était comparé ? Nombreux sont les critiques à avoir rapproché The Broom of the System (1987) de The Crying of Lot 49 (1966), ou qualifié Infinite Jest de second Gravity's Rainbow (1973).

14 Tore Andersen a proposé une première réponse à cette question. Sa contribution a en effet montré que les incessantes comparaisons à Pynchon étaient en partie à mettre sur le compte de stratégies éditoriales régissant présentations et quatrièmes de couverture. Un exemple particulièrement révélateur a été celui de la critique de The Broom of the System par Michiko Kakutani du New Yorker, dont Tore Andersen a montré qu'elle reprenait quasiment verbatim la quatrième de couverture de l'édition Viking par endroits. Sa communication a soutenu que le paratexte avait durablement joué le rôle d'œillères, en escamotant la puissante influence de DeLillo sur les écrits de Wallace. L'intervention s'est conclue sur le fait que depuis la mort de Wallace en 2008, les références à Pynchon étaient entièrement absentes du paratexte wallacien (pour This is Water (2009), The Pale King (2011) ou Both Flesh and Not (2012))

\section{Lee Konstantinou (University of Maryland, USA), «What is a Turdnagel? »}

Lee Konstantinou a proposé une réévaluation de la place de la philosophie dans l'œuvre de Wallace. Sa communication s'est ouverte sur une exploration de la portée insoupçonnée du néologisme wallacien: «turdnagel». Dans The Pale King (2011), le terme désigne une catégorie très particulière d'employés de l'Internal Revenue Service qui forment un cercle fermé et mystérieux. S'appuyant sur une analyse attentive des carnets de notes et brouillons du Pale King déposés au Harry Ransom Center, Lee Konstantinou a montré que le terme «turdnagel» était en fait une référence au philosophe américain Thomas Nagel.

Ses réflexions sur le caractère subjectif de l'expérience, telles qu'elles sont exposées dans son célèbre essai «What Is it Like To Be a Bat?» (1974) notamment, rejoignent certains thèmes centraux de The Pale King: l'irréductibilité du solipsisme et la transformation de la personne en personnage. Dès lors, la communication revenait sur la thèse communément admise selon laquelle seuls les premiers écrits de Wallace 
dialoguaient ouvertement avec la philosophie. En retraçant l'influence de la pensée de Thomas Nagel sur l'œuvre qui a occupé les dix dernières années de la vie de Wallace, Lee Konstantinou a proposé une révision en profondeur de ce paradigme.

17 Les actes du colloque accompagneront la publication française d'Infinite Jest par les Éditions de l'Olivier à la rentrée 2015.

18 Lien vers le programme du colloque :

19 http://www.infinitewallace.org/program/

20 Lien vers les résumés des interventions :

21 http://www.infinitewallace.org/abstracts/

\section{NOTES}

1. Les intervenants, par ordre d'intervention, étaient: Bart Thornton; Mike Miley; Tony McMahon; Jay Johnson; Marshall Boswell; Tim Groenland; Jackie O’Dell; John Roache; Laura Morris; Laura Kreyder; Calvin Thomas; Stephanie Lambert; Lefteris Kalospyros; Kostas Kaltsas; Tore Andersen; Daniel Mattingly; Pater Waldstein; Christopher Kocela; Jason Ford; Jeffrey Fisher; Jurrit Daalder; David Hering; Bill Lattanzi; JT Jackson; Michell Cunningham; Jacopo Cozzi; Hadrien Laroche; Lee Konstantinou; Allard Den Dulk; Anthony Leaker; Franz Kaltenbeck; Robert McLaughlin; Toon Staes; Ralph Clare; Mary Holland; Adam Kelly.

\section{AUTHOR}

ARIANE MAK

EHESS 